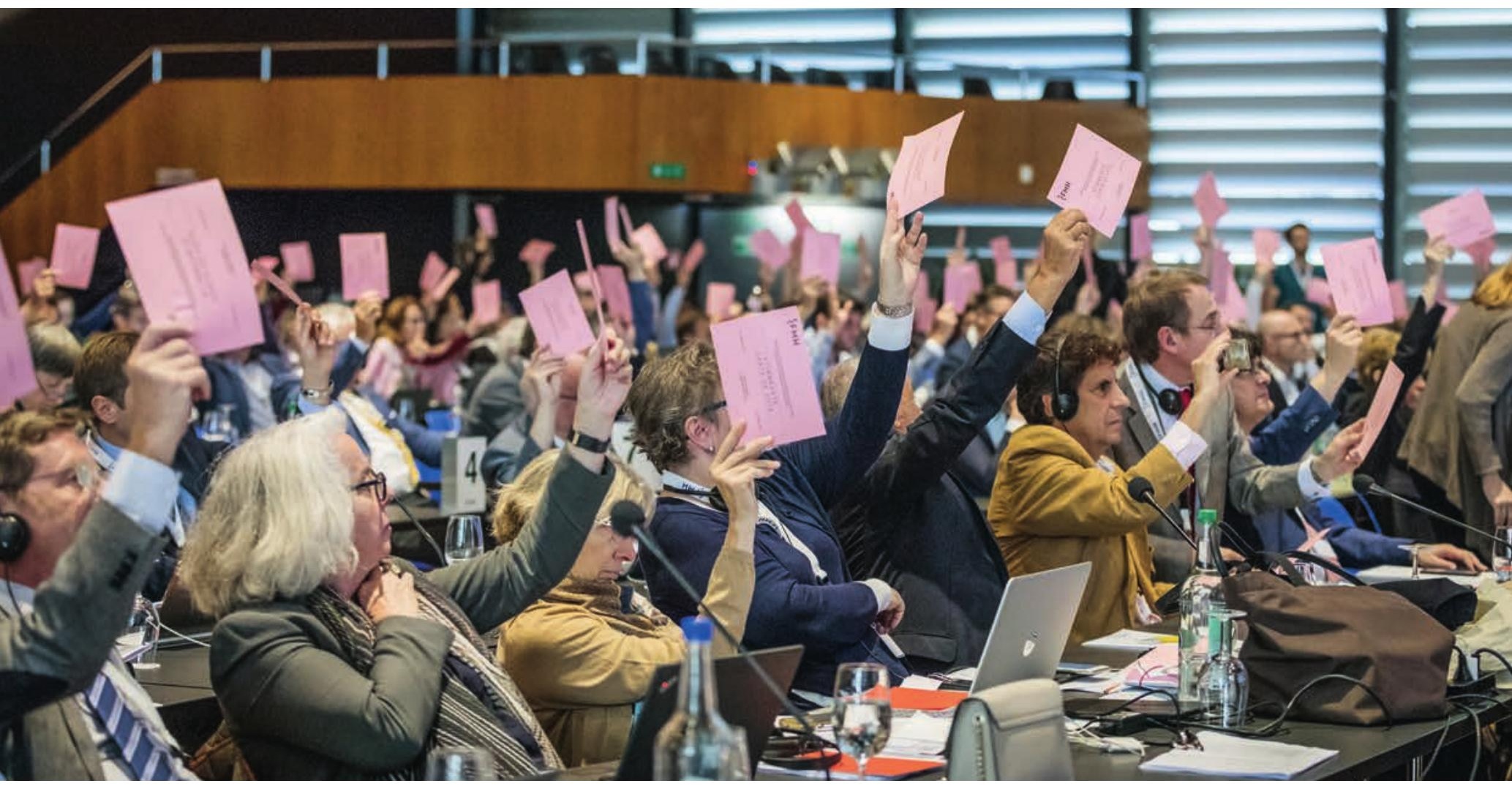

Les délégués ont approuvé à une écrasante majorité la structure des prestations actualisée du tarif ambulatoire et la recherche de solutions communes avec les partenaires tarifaires.

\title{
Procès-verbal décisionnel de la deuxième Chambre médicale 2018
}

\section{Monika Henzen}

Cheffe de la division Secrétariat central

Remarque de la rédactrice: pour faciliter la lecture de ce procès-verbal, les points sont indiqués selon leur numérotation et non l'ordre de la discussion.

\section{Message de bienvenue et informations}

Une fois le quorum atteint, le Dr méd. Jürg Schlup/président de la FMH ouvre la deuxième Chambre médicale 2018. Il souhaite la bienvenue à tous les délégués et en particulier à la nouvelle secrétaire générale, $D r$ iur. Ursina Pally-Hofmann. Elle a repris la direction opérationnelle du Secrétariat général de la FMH tout en assumant la fonction de cheffe de la division Service juri- dique. Cette Grisonne de 50 ans est docteur en droit, avocate et sage-femme.

Après les annonces d'ordre organisationnel par Ursina Pally Hofmann/secrétaire générale de la FMH, le bureau est désigné. Il est composé $d u$ président, des viceprésidents, de la secrétaire générale, de Me Anne-Sylvie Thiébaud/responsable du bureau électoral, de Me Julien Duruz/suppléant du bureau électoral et des scrutateurs suivants élus par 110 voix pour, 2 contre et 0 abstention: Alexander Zimmer, Christoph Helbling, Jean-Pierre Grillet, Florian Leupold, Hans-Anton Vogel, Franziska Zogg, Lucia Zürcher, Pierre Arnold, Karl-Olof Lövblad, Anja Zyska Cherix. 


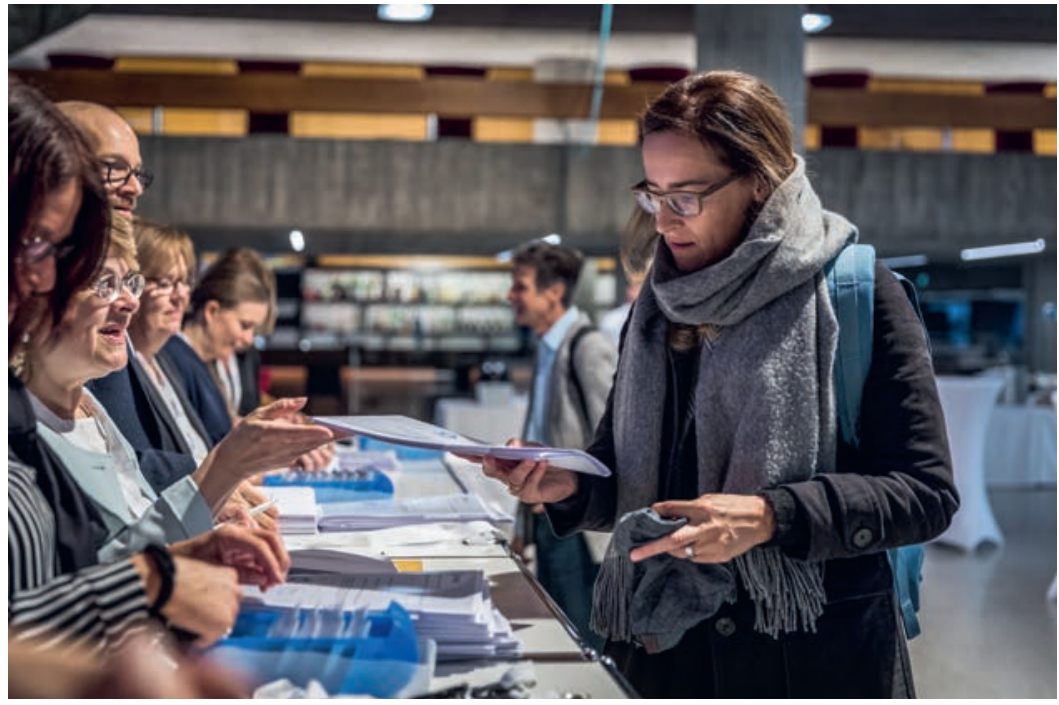

Les participants à la Chambre médicale sont accueillis avec le sourire par les collaboratrices et collaborateurs de la FMH qui leur transmettent les documents de séance.

\section{Ordre du jour}

Proposition:

Approbation de l'ordre du jour.

\section{Décision:}

L'ordre du jour est approuvé à l'unanimité.

Conformément à l'art. 11 al. 3 RE, la Chambre médicale fixe au début de chaque séance l'heure limite à partir de laquelle elle ne peut plus prendre de décision ni procéder à des élections. Le président propose de clore la séance à $18 \mathrm{hoO}$.

Proposition pour l'heure de fin de séance:

La fin de la séance est fixée à $18 \mathrm{~h} 00$. Après $18 \mathrm{ho0}$, aucune décision ne pourra être prise ni aucune élection effectuée.

\section{Décision:}

La proposition est adoptée par 110 oui, 0 non et 3 abstentions.

Le président soumet deux autres motions d'ordre concernant la limitation du temps de parole et le dépôt des propositions.

Conformément à l'art. 11 al. 7 RE, le temps accordé à chaque orateur peut être limité sur proposition de la Chambre médicale ou du président. Compte tenu du nombre des objets à l'ordre du jour et du temps à disposition, il est proposé de limiter le temps de parole à deux minutes.

$1^{\text {re }}$ motion d'ordre-Limitation du temps de parole:

Pour le traitement des points à l'ordre du jour de la Chambre médicale de ce jour, le temps de parole est limité à deux minutes par orateur. Aucune limite ne s'applique au président de chacune des organisations médicales ayant droit de vote ou de proposition ou à son suppléant, ni à l'intervenant du Comité central.

\section{Décision:}

La proposition est adoptée par 105 oui, 3 non et 1 abstention.

L’art. 11 al. 5 RE prévoit que les délégués, les membres du Comité central et le secrétaire général ont chacun le droit de faire des propositions quant aux objets de l'ordre du jour soumis à la discussion. Le texte des propositions doit être présenté au président par écrit ou exceptionnellement par oral, avant ou pendant la séance.

$2^{\mathrm{e}}$ motion d'ordre - Dépôt par écrit des propositions: Les amendements et propositions doivent être présentés au président par écrit. Les propositions présentées par oral sont invalides et ne seront pas acceptées.

Décision:

La proposition est adoptée par 100 oui, 23 non et 8 abstentions.

\section{Allocution d'ouverture du président}

Dans son étude «Structure d'approvisionnement ambulatoire et analyse des besoins en Suisse» publiée en septembre 2018, santésuisse postule un surapprovisionnement de médecins en Suisse. Sur la base de ses projections pour l'année 2030, santésuisse prévoit une nette tendance vers un surapprovisionnement dans toute la Suisse. La réalité est pourtant différente. Les postes vacants ont augmenté au cours des trois dernières années. Fin 2017, il y avait nettement plus de postes de médecins vacants (environ 1400) que de médecins ayant obtenu leur diplôme pendant la même année en Suisse. La pénurie de médecins perdure.

La prise en charge ambulatoire a considérablement changé. A l'augmentation des maladies chroniques et du nombre de patients polymorbides se sont ajoutées l'évolution démographique mais aussi l'offre insuffisante de places d'études ces 20 dernières années, qui se répercute sur la situation actuelle. Le fardeau administratif excessif et, depuis peu, la limitation de la durée des consultations réduisent le temps disponible pour la prise en charge médicale des patients.

La Suisse a besoin de médecins avec des diplômes étrangers et elle est reconnaissante de leur présence. Pourtant, le Conseil fédéral veut durcir le pilotage des admissions. La FMH exige une admission selon des critères de qualité avec trois ans d'activité médicale dans la discipline demandée pour l'admission dans un établissement de formation postgraduée suisse reconnu 


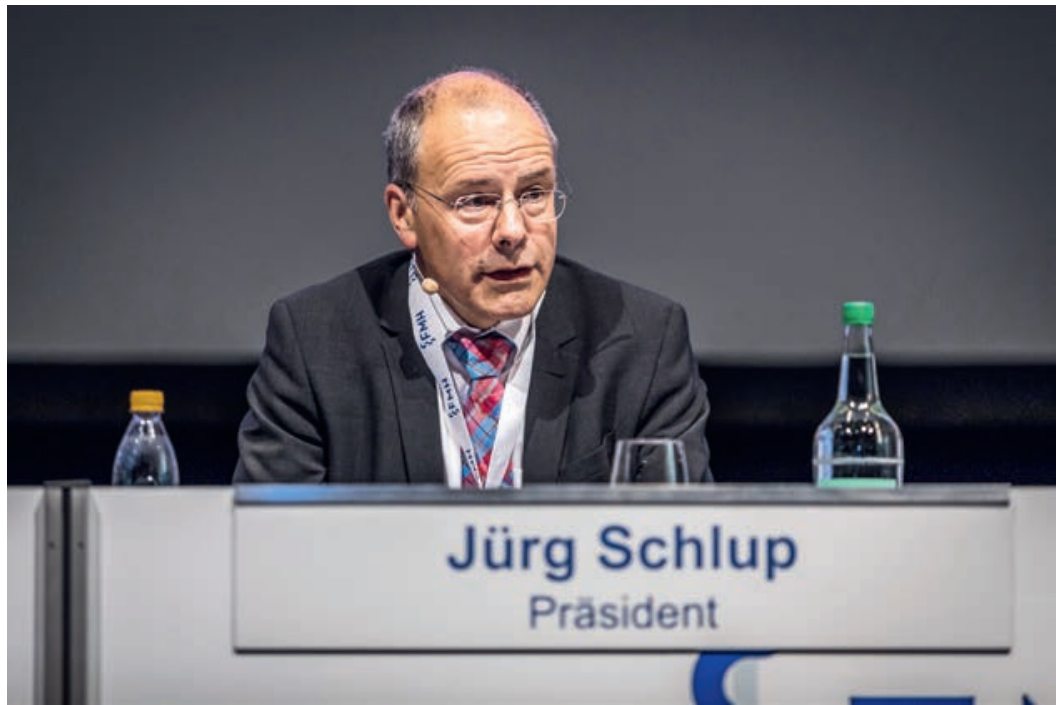

Le Dr Jürg Schlup, président de la FMH, dirige la séance.

et l'attestation des compétences linguistiques requises pour exercer dans la langue de la région choisie. Parallèlement, un changement culturel est en marche dans la population. Les attentes des patients augmentent, les assurés souhaitent aujourd'hui un traitement rapide et complet. Pour y répondre, les politiques exigent des mesures pour freiner l'augmentation des coûts avec des plafonds et un budget global. Les coûts n'ont baissé dans aucun des pays qui appliquent un budget global. Une telle mesure conduit plutôt à des temps d'attente, à une médecine à deux vitesses et à des tâches administratives disproportionnées et excessives pour répartir le budget. Exiger un budget glo-

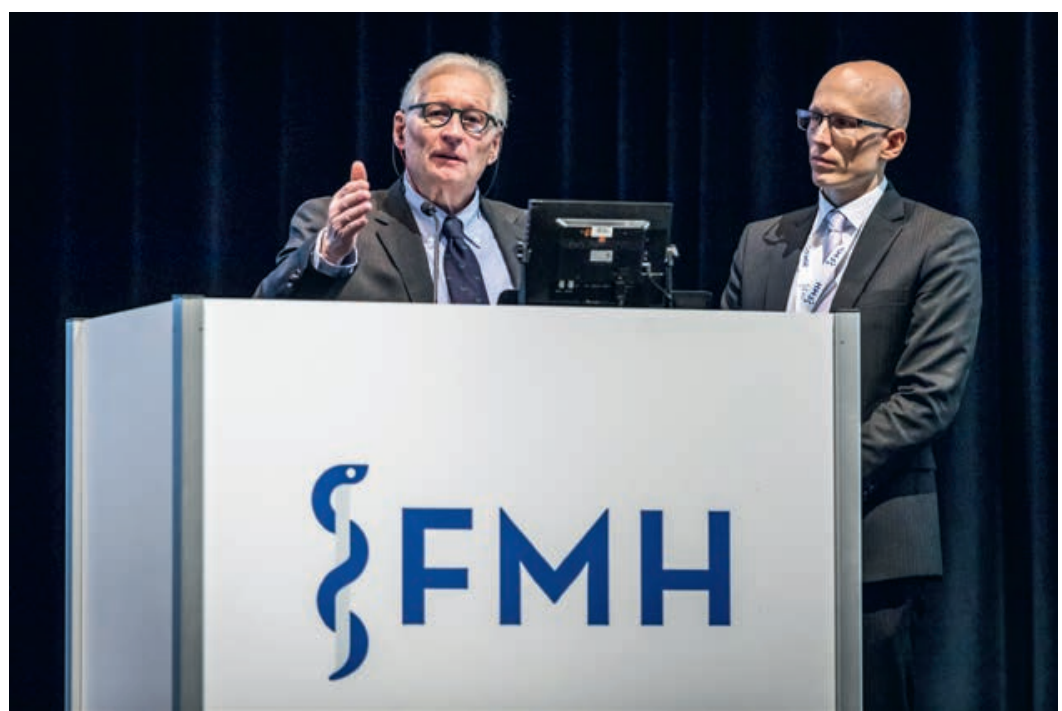

Le Dr Urs Stoffel, membre du Comité central de la FMH et responsable du département Médecine et tarifs ambulatoires, et Patrick Müller, chef de la division Médecine et tarifs ambulatoires, présentent la structure des prestations actualisée du tarif ambulatoire. bal, c'est accepter une médecine à deux vitesses. Le corps médical ne veut ni de médecine à deux vitesses ni de temps d'attente. Au contraire, il veut la réduction des tâches administratives et un financement uniforme des prestations ambulatoires et stationnaires qui permettraient d'économiser des coûts. Et le corps médical veut un système tarifaire approprié.

Le blocage se poursuit dans le système de santé et la pression politique s'accroît. Le corps médical doit transformer en opportunités les défis lancés au niveau politique. Dans un système politique démocratique, la politique c'est débattre. La meilleure des solutions n'a aucune chance si on refuse le débat. La politique, ce n'est pas seulement le destin comme le disait Napoléon, mais c'est aussi la solution. Pour recouvrer l'autonomie tarifaire, le président en appelle à tous les délégués et leur demande de poursuivre avec détermination leur engagement pour la révision en cours du tarif ambulatoire. Après plusieurs années de travail, la FMH a développé et élaboré une structure des prestations dont elle peut être fière.

L'association des médecins a besoin de soutien politique dans les parlements cantonaux et les Chambres fédérales. Lors des élections fédérales 2019, elle recommande donc de soutenir les candidats qui, d'une part, s'engagent pour les positions du corps médical - notamment le refus du budget global, le soutien du libre choix du médecin, l'autonomie tarifaire et un pilotage des admissions au moyen de critères de qualité - et qui, d'autre part, ont des chances d'être élus ou réélus.

Le président remercie tous les collègues pour leur travail quotidien au service des patients et de la population.

\section{Tarifs}

2.1 Révision du tarif ambulatoire: adoption de la structure des prestations négociée, y c. les modèles de coûts et les points tarifaires Dr méd. Urs Stoffel/membre du Comité central et responsable du département Médecine et tarifs ambulatoires informe de l'état actuel de la révision du tarif médical ambulatoire. Il rappelle la décision de la Chambre médicale du 2 mai 2018 par laquelle les délégués ont approuvé à l'unanimité la proposition de la FMH (projet TARCO) pour la structure des prestations comme base essentielle d'un tarif approprié et calculé selon les principes de l'économie d'entreprise. Conformément à la décision du Cockpit, les négociations avec les partenaires tarifaires au sein de l'ats-tms SA se sont déroulées en parallèle à l'élaboration de la structure des prestations afin de pouvoir terminer la révision à temps. Les négociations menées jusqu'ici avaient pour axes 


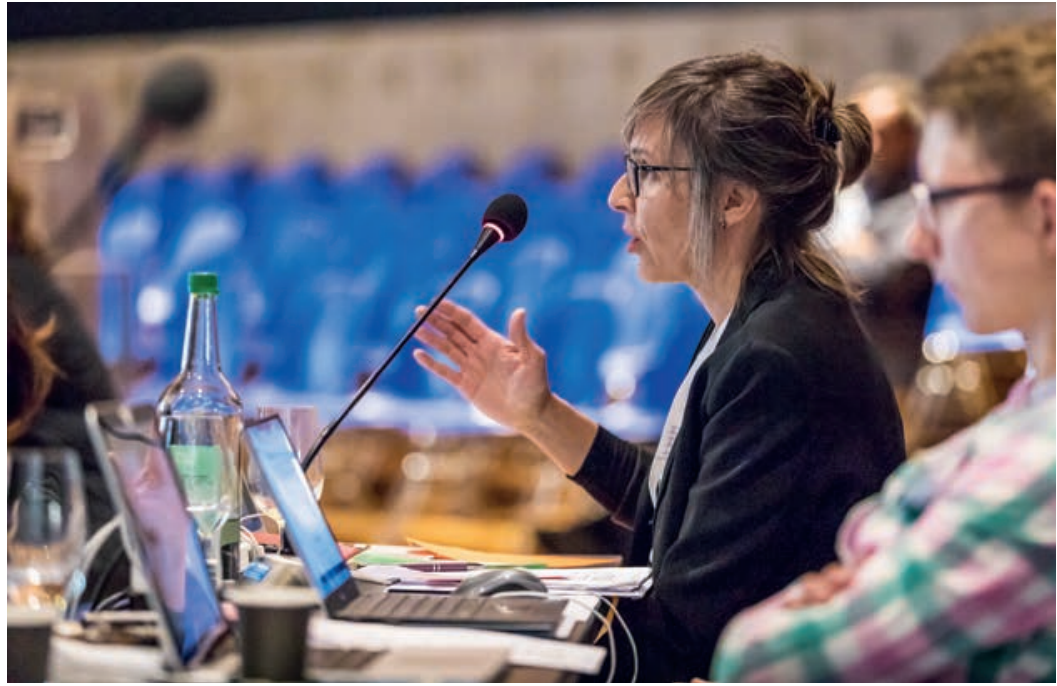

Les délégués représentent leurs organisations de base et de discipline à la Chambre médicale et s'engagent dans le débat sur différents thèmes et propositions: ici, la proposition de I'ASMAC. l’assemblée générale de mars 2018. La grande majorité des adaptations proposées par la FMH a été mise en œuvre. C'est un geste positif des partenaires tarifaires en faveur de la FMH. Le 20 septembre 2018, le comité de $\mathrm{H}+$ a décidé à l'unanimité de résilier la convention d'actionnaires de la société ats-tms SA du 26 mars 2018. La FMH, curafutura et la CTM restent cependant dans la société ats-tms SA.

Les travaux de la $1^{\text {re }}$ étape sur la structure des prestations actuelles, y compris les modèles de coûts et les points tarifaires, sont terminés et ne seront en substance plus modifiés (excepté les corrections de l'orthographe, les traductions en français et en italien, les compléments dans les groupes de prestations, la structure des chapitres et des positions tarifaires ou les corrections d'erreurs manifestes). Après approbation par la Chambre médicale, les résultats obtenus seront communiqués au grand public et aux milieux politiques comme premier succès. La structure des prestations négociée, y compris les points tarifaires, constitue le maillon central de la structure tarifaire révisée. Les points encore en suspens concernant les règles de facturation et le transcodage seront abordés dès la mi-novembre 2018.

Lors de la $2^{\mathrm{e}}$ étape, les règles de facturation seront négociées pour chaque chapitre en collaboration avec les sociétés de discipline concernées. Le transcodage et les simulations seront préparés en parallèle. Les travaux de la $2^{\mathrm{e}}$ étape seront achevés en mars 2019. Ensuite, la structure tarifaire totalement révisée, y c. les règles de facturation remaniées, sera soumise une nouvelle fois aux organes internes de la FMH (Assemblée des délégués et Chambre médicale) sous forme d'un ensemble global incluant les conventions de base et les autres concepts (p. ex. contrôle de la plausibilité des prestations à l'acte).

La FMH a déposé une proposition d'adaptation de la désignation de la nouvelle proposition tarifaire au conseil d'administration d'ats-tms SA. Le titre de travail provisoire est «Structure des prestations ambulatoires». Après des analyses et calculs détaillés au sujet de la valeur intrinsèque quantitative, la direction du groupe de travail sur les valeurs intrinsèques est parvenue à la conclusion que la valeur intrinsèque médicale individuelle (VIMI) ne pouvait plus être justifiée sur la base des données actuelles. Le Cockpit a suivi la proposition de la direction du groupe de travail de renoncer pour l'heure à négocier la VIMI.

Selon Urs Stoffel/membre du Comité central et responsable du département Médecine et tarifs ambulatoires, la structure des prestations présentée n'est pas encore parfaite et contient des compromis qu'il a fallu accepter lors des négociations. Cela peut poser problème 
pour certaines sociétés de discipline mais les discussions à ce sujet seront menées lors de la $2^{\mathrm{e}}$ étape.

L'approbation interne de l'ensemble de la structure tarifaire par les organes de la FMH est prévue en avril/ mai 2019, et le dépôt définitif au Conseil fédéral en juin 2019. Le tarif révisé pourrait donc entrer en vigueur le $1^{\mathrm{er}}$ janvier 2020.

Urs Stoffel/membre du Comité central et responsable du département Médecine et tarifs ambulatoires revient sur les mesures visant à maîtriser les coûts mentionnées par le président dans son allocution d'ouverture. Elles seront radicales si les partenaires tarifaires n'arrivent pas à se mettre d'accord. Une des mesures mises en consultation concerne la création d'une organisation tarifaire nationale pour le secteur ambulatoire. Le Conseil fédéral peut définir des principes concernant la forme et l'exploitation de l'organisation dans une ordonnance. Si les partenaires tarifaires n'arrivent pas à se mettre d'accord sur la mise en place d'une organisation tarifaire nationale dans les deux ans à compter de l'entrée en vigueur, le Conseil fédéral interviendra et instituera une organisation tarifaire nationale pour le secteur ambulatoire.

Dans la discussion qui suit, les délégués se déclarent clairement favorables à un renforcement des soins ambulatoires. Il est rappelé que cela passe par une tarification appropriée des prestations ambulatoires. Pour la FMH, les valeurs clés des négociations sont désormais définies et les différentes sociétés de discipline seront sollicitées pour la finalisation de leurs chapitres.

Le Comité central soumettra la structure tarifaire définitive à l'approbation des délégués lors de la Chambre médicale du 9 mai 2019.

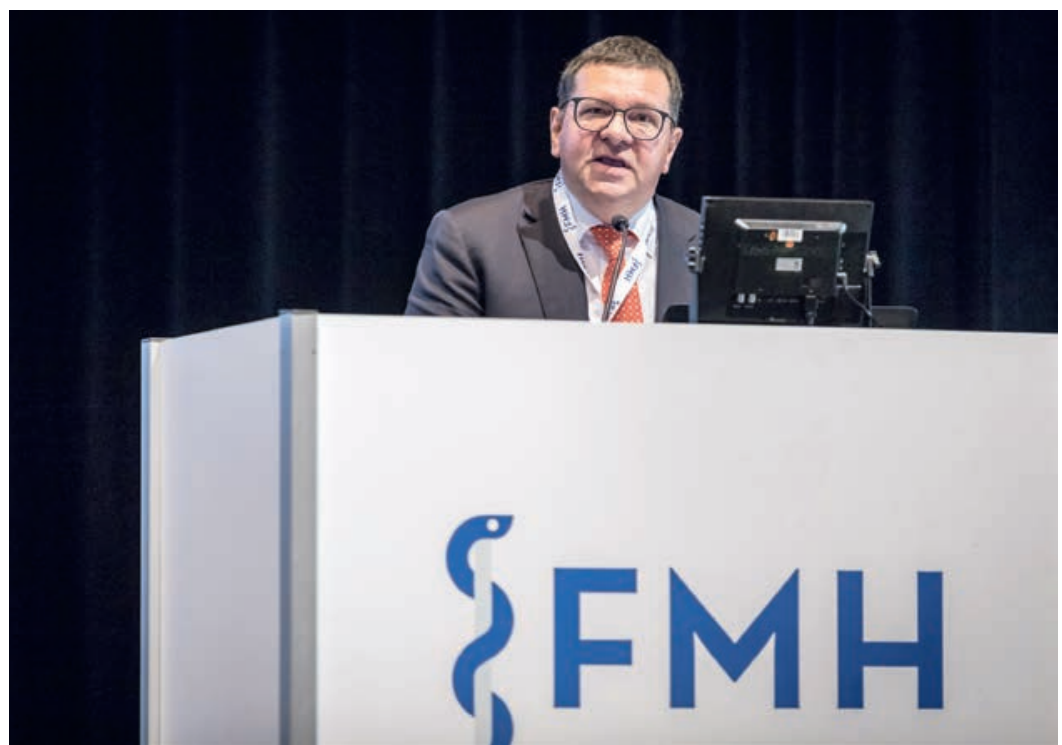

Reto Heiz, chef a.i. de la division Administration et finances, présente le budget 2019 de la FMH et de la CdG.
Le président soumet au vote les modalités de vote.

Proposition du Comité central:

Un vote global sur les trois propositions versus trois votes distincts.

\section{Décision:}

150 délégués se prononcent pour un vote global sur toutes les propositions; 2 délégués pour trois votes. 1 délégué s'abstient. Les trois propositions sont donc votées ensemble.

Proposition du Comité central:

La Chambre médicale approuve

- définitivement la structure des prestations négociée (nomenclature) présentée incluant les modèles de coûts et les points tarifaires (conformément au navigateur tarifaire «structure des prestations ambulatoires» V1.0) en tant que résul-

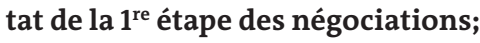

- le dépôt de la structure des prestations négociée (nomenclature) incluant les modèles de coûts et les points tarifaires (conformément au navigateur tarifaire «structure des prestations ambulatoires» V1.0) pour un examen préalable par le Conseil fédéral et l'OFSP;

- la marche à suivre pour les négociations avec les partenaires tarifaires en vue de l'élaboration des règles de facturation tarifaires, des conventions, du transcodage et des simulations en tant que résultat de la $2^{\mathrm{e}}$ étape. Ce résultat sera présenté aux organes d'approbation internes de la FMH (Assemblée des délégués et Chambre médicale) en avril/mai 2019.

Décision:

Les trois propositions sont adoptées par 158 oui, 1 non et 3 abstentions.

Urs Stoffel/membre du Comité central et responsable du département Médecine et tarifs ambulatoires remercie toutes les personnes impliquées dans le projet de même que toute son équipe à Olten pour leur inlassable et formidable engagement.

\section{Budgets 2019}

Jürg Schlup/président de la FMH souhaite la bienvenue à Reto Heiz, chef par intérim de la division Administration et finances du Secrétariat général depuis le $1^{\mathrm{er}}$ septembre 2018.

Avant l'examen du budget, le président informe que deux propositions ont été déposées pour ce point à l'ordre du jour. Dans sa proposition déposée le 4 octobre 2018, la SSMIG demande le prélèvement d'une contribution exceptionnelle en faveur de l'initiative 


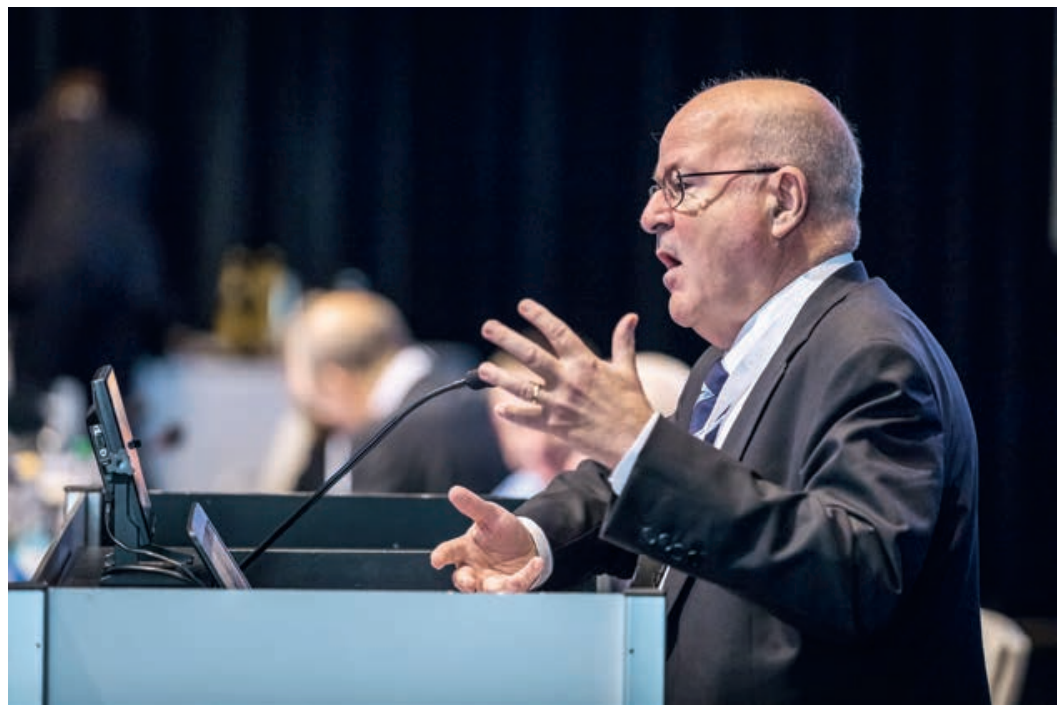

Le Dr Werner Bauer, président de I'ISFM, présente le budget 2019 de I'ISFM.

"Oui à la protection des enfants et des jeunes contre la publicité pour le tabac». Avec sa proposition déposée aujourd'hui, l'ASMAC souhaite clarifier le financement des interventions politiques à l'avenir. L'ASMAC est d'accord pour que sa proposition ne soit traitée qu'après le vote du budget consolidé.

\subsection{Budget 2019 de l'ISFM}

Werner Bauer/président de l'ISFM rend compte de l'accréditation 2018 qui est désormais achevée et a permis aux experts de tirer un bilan positif de la qualité des cursus de formation en médecine humaine. Placés sous la responsabilité de l'ISFM et des sociétés de discipline médicale, ces cursus sont donc accrédités pour une nouvelle période de sept ans. La procédure est longue et coûteuse puisque l'ISFM prend en charge les coûts des sociétés de discipline médicale. Les rares conditions et recommandations émises seront discutées avec les sociétés concernées. Pour la formation continue, l'OFSP) réfléchit à un engagement plus régulateur et suggère la création d'un groupe thématique chargé d'examiner les éventuels besoins d'intervention. L'OFSP envisage p. ex. une formation continue plus structurée et mieux contrôlée, voire une recertification tous les 10 ans. L'ISFM considère cependant que la formation continue relève de la responsabilité du corps médical et que c'est à lui de l'organiser. La plateforme "Avenir de la formation médicale» planche actuellement sur la question de savoir à quelle fréquence et pourquoi la formation postgraduée dure plus longtemps que celle prévue dans les programmes de formation et a mandaté une étude à ce sujet. Le groupe thématique "Coordination de la formation médicale postgraduée» a pour l'instant achevé ses travaux. L'Ob- servatoire suisse de la santé (Obsan) a élaboré un modèle permettant d'anticiper le nombre et le besoin de médecins spécialistes en Suisse; compte tenu des données actuellement insuffisantes, ce modèle a encore besoin d'être amélioré afin d'être véritablement pertinent.

Christoph Hänggeli/directeur de l'ISFM informe de la situation financière de l'ISFM. Avec un investissement annuel d'un million de francs, le logbook électronique 2.0 représente le poste budgétaire le plus important. Les coûts du projet sont respectés, mais l'introduction du nouveau tableau de bord accuse un léger retard. Le "cockpit» devrait être disponible au premier trimestre 2019. Le budget 2019 prévoit un bénéfice de 2000 francs. Cet équilibre budgétaire est principalement lié à la hausse du nombre de titres de spécialiste décernés mais aussi à diverses augmentations de taxes. Les établissements de formation postgraduée participeront davantage aux dépenses de l'ISFM par des taxes de certification et taxes de visite. L'obtention du deuxième et de tous les titres suivants de spécialiste sera soumise à une taxe de 2000 francs. La taxe à payer pour le premier titre de spécialiste demeure inchangée (4000 francs depuis 2002).

Côté dépenses, la promotion de projets est réintroduite avec un montant de 150000 francs pour récompenser des projets innovants dans le domaine de la formation postgraduée. Par ailleurs, des coûts supplémentaires sont à prévoir en 2019 pour les projets électroniques et le personnel. Au $1^{\text {er }}$ janvier 2020, la fortune devrait s'élever à 2,2 millions de francs.

Selon la planification financière 2020-2021, l'ISFM table sur une croissance des bénéfices et une progression de la fortune qui dépassera à nouveau la barre des 3 millions de francs.

\subsection{Budget 2019 de la FMH}

Reto Heiz/chef a. i. de la division Administration et finances détaille le budget 2019 de la FMH qui prévoit un déficit de 144000 francs.

Le frein aux dépenses introduit dès le budget 2016 et accepté par la Chambre médicale a été respecté et sera maintenu. Le budget 2019 a également bénéficié de l'impact positif des mesures de stabilisation budgétaire mises en place jusqu'ici.

Les pertes minimes résultent entre autres de l'accroissement des frais de consultation IT et des développements de logiciels. Les frais de personnel restent inchangés tandis que les salaires de tiers et d'experts sont moins élevés. En revanche, les coûts de projets structurels comme "I-Governance», la rénovation totale des locaux de l'Elfenstrasse et le monitorage du projet TARCO augmenteront ces prochaines années, ce 
qui affectera la sécurité de la planification financière. Le nombre croissant de membres comme la réduction des contributions exceptionnelles décidées par la Chambre médicale n'arrivent de justesse pas à couvrir les dépenses.

Le budget 2019 démontre que la planification financière à moyen terme permet à la FMH de discipliner ses dépenses.

\subsection{Budget 2019 de la CdG}

Reto Heiz/chef a. i. de la division Administration et finances présente le budget de la CdG qui reste modeste avec un montant de 75000 francs. La CdG contribue, elle aussi, à mettre en œuvre les mesures de stabilisation budgétaire adoptées.

\section{Rapport de gestion de la CdG}

Dr méd. Adrian Sury/président de la CdG rend compte des séances de l'année, consacrées notamment à la rénovation totale des locaux de l'Elfenstrasse et à la situation financière de la FMH. Après avoir pris connaissance du projet détaillé des travaux de rénovation, la CdG s'inquiète de l'ampleur des coûts. Elle a déjà suggéré au Comité central de soumettre ce budget de rénovation une nouvelle fois au vote des délégués de la Chambre médicale.

La CdG s'est longuement penchée sur le budget de la FMH et se réjouit du tournant pris par l'ISFM. Le budget consolidé de la FMH présente une perte au bilan de 144000 francs. Les charges salariales ont été budgétisées à un montant supérieur de 1854000 francs par rapport aux comptes 2017. Cette différence est liée au recrutement de nouvelles personnes à l'ISFM et au Secrétariat général. Le taux de fluctuation du personnel, actuellement élevé, rend difficile d'estimer l'évolution. Les provisions correspondent aux besoins et les liquidités sont excellentes. Le rendement des placements est inférieur à l'excellent résultat de 2017. Mais la réorientation de la stratégie de placement avec moins d'obligations et une meilleure gestion des placements en actions a permis d'éviter un bien plus mauvais résultat.

L'effet positif des mesures de stabilisation budgétaire mises en œuvre jusqu'ici est également réjouissant. En tant qu'organe de surveillance de la FMH, la CdG recommande aux délégués de voter le budget de la FMH et de l'ISFM. Adrian Sury/président de la CdG remercie le Comité central, le Secrétariat général, le Comité et la Direction de l'ISFM pour leur collaboration transparente et leur excellente culture du dialogue.

Jürg Schlup/président de la FMH souligne que les craintes de la CdG concernant la rénovation complète des locaux de l'Elfenstrasse sont actuellement injustifiées.
Le membre du Comité central en charge de ce projet, Christoph Bosshard, informera ce jour de l'état actuel du projet de rénovation et des prochaines étapes. Les délégués seront informés en détail de la planification et du total des coûts prévus par les maîtres d'œuvre lors de la Chambre médicale du 9 mai 2019.

\section{Contribution spéciale NAKO} (organe de consolidation nationale) Selon Urs Stoffel/membre du Comité central et responsable du département Médecine et tarifs ambulatoires, la collecte des données par les médecins en cabinet est indispensable et jouera un rôle de plus en plus important pour garantir la parité des données avec les partenaires tarifaires. La FMH est tributaire de ces données pour poursuivre la révision tarifaire avec l'étape $2 \mathrm{du}$ projet TARCO et pour assurer le monitorage exigé par l'OFSP. La contribution spéciale sert au financement de base de l'infrastructure et au bon fonctionnement de NewIndex SA, et permet ainsi au corps médical d'avoir accès au système NAKO, grâce auquel il peut procéder à des analyses et évaluations ciblées de données.

\section{Proposition du Comité central:}

La Chambre médicale approuve la contribution spéciale 2019 en faveur de NAKO à hauteur de 40 francs pour les catégories 1 et 2 .

Décision:

La proposition est adoptée par 139 oui, 7 non et 14 abstentions.

\section{Contribution spéciale pour la Revue médicale suisse}

Reto Heiz/chef a. i. de la division Administration et finances propose à la Chambre médicale d'approuver la contribution spéciale de 10 francs en faveur de la Revue médicale suisse pour 2019.

Proposition du Comité central:

La Chambre médicale approuve la contribution spéciale 2019 en faveur de la Revue médicale suisse de 10 francs pour les catégories 1, 2, 3, 5 et 6 . Décision:

La proposition est adoptée par 147 oui, 3 non et 8 abstentions.

\section{Contribution exceptionnelle SSMIG}

Philippe Luchsinger/SSMIG motive la proposition déposée le 4 octobre 2018 pour une contribution de soutien de 5 francs en faveur du "Oui à la protection des enfants et des jeunes contre la publicité pour le tabac». L'objectif de collecter le plus rapidement possible de nombreuses signatures dans le délai imparti n'a pas 
été atteint. Comme il s'agit ici d'une initiative importante également pour la FMH, il propose de prendre toutes les mesures nécessaires pour récolter le nombre de signatures prévues. Dans la constellation actuelle, la loi sur les produits du tabac va devenir un instrument inefficace - notamment en matière de prévention - qui ouvrira grand la porte au travail des lobbyistes. Il faut absolument l'empêcher. Il regrette l'absence de mobilisation de la part du corps médical, mais reste convaincu qu'un soutien professionnel permettra de collecter les signatures manquantes. Une contribution exceptionnelle enverrait un signal positif.

Proposition de la SSMIG:

Prélever une contribution exceptionnelle en 2019 en faveur du «Oui à la protection des enfants et des jeunes contre la publicité pour le tabac» de 5 francs pour les catégories $1,2,3,4,5,6,7$ et 11 . Décision:

La proposition est rejetée par 90 non, 70 oui et 6 abstentions.

Proposition de l'ASMAC sur le financement des interventions politiques

Anja Zyska Cherix/présidente de l'ASMAC souligne que son association soutient la proposition de la SSMIG tout comme l'initiative populaire «Oui à la protection des enfants et des jeunes contre la publicité pour le tabac». Les délégués de l'association ont cependant un peu de mal avec la démarche choisie par la SSMIG qui soulève des questions de fond. Comment les initiants veulent-ils utiliser les moyens supplémentaires? S'agit-il d'un cas particulier ou va-t-il faire école? Concernant cette seconde question, l'ASMAC propose de réfléchir à une nouvelle manière de traiter les demandes de financement liées à des interventions politiques et de trouver une solution valable pour tous. Des propositions comme celle de la SSMIG deviendraient ainsi superflues à l'avenir.

\subsection{Cotisations de membres}

Reto Heiz/chef a. i. de la division Administration et finances présente les cotisations de membres pour 2019. Les montants de base restent inchangés par rapport à l'année précédente.

La Chambre médicale approuve les cotisations de membres suivantes pour 2019:

Catégories 1 et 2: CHF 760

Catégorie 3: $\quad$ CHF 485

Catégorie 4: $\quad$ CHF 355

Catégorie 5: $\quad$ CHF 294

Catégorie 6: $\quad$ CHF 188

Catégorie 7: $\quad$ CHF 142

Catégorie 11: $\quad$ CHF 178
Proposition du Comité central:

Approbation des cotisations de membres 2019.

\section{Décision:}

Les cotisations de membres 2019 sont approuvées par 162 oui, 0 non et 2 abstentions.

\subsection{Budget 2019 consolidé}

Proposition du Comité central:

La Chambre médicale adopte le budget 2019 consolidé de la FMH qui présente une perte de kCHF 144 (gain de l'ISFM à hauteur de kCHF 2 et perte de la FMH à hauteur de kCHF 146).

Décision:

Le budget 2019 consolidé de la FMH est adopté par 163 oui, 0 non et 5 abstentions.

\section{Proposition de l'ASMAC}

Proposition de l'ASMAC:

Le Comité central de la FMH présente à la Chambre médicale du printemps 2019 une proposition pour financer les demandes de soutien à des interventions politiques via les fonds existants de la FMH. Décision:

La proposition est adoptée par 119 oui, 37 non et 6 abstentions.

\section{Rapport 2018 sur les mesures de stabilisation budgétaire}

Reto Heiz/chef a. i. de la division Administration et finances livre un tour d'horizon des résultats réalisés au $1^{\mathrm{er}}$ semestre 2018. Les objectifs fixés par la Chambre médicale le 30 octobre 2014 visaient à atteindre l'équilibre financier entre les dépenses effectives et les recettes générées afin que la FMH puisse se concentrer sur ses activités clés et développer des tâches secondaires. Seule une situation financière équilibrée permettra à la FMH d'être perçue comme un partenaire important de la santé et de se concentrer sur son cœur de métier. Les objectifs d'économies de 5,8 millions de francs au total devaient être atteints en deux étapes entre 2017 et 2019; d'une part par une baisse des dépenses de 3,8 millions de francs, d'autre part par une augmentation des recettes de 2 millions de francs. A la fin décembre 2017, $83,8 \%$ de ces objectifs ont été réalisés.

Le premier semestre 2018 fait état d'un résultat négatif de kCHF 366, soit moins que prévu initialement. Les différences principales s'expliquent d'une part par les retards dans la réalisation de projets et par les mesures qui n'ont pas été entièrement mises en œuvre dans le domaine des «organes». Comme il s'agit d'un bilan intermédiaire et que les différentes mesures ne pourront être précisément chiffrées qu'à la fin de l’année fiscale, 
le résultat de l'évaluation du bilan annuel 2018 devrait être plus explicite. Avec le frein aux dépenses approuvé par la Chambre médicale en 2014, la FMH entend poursuivre sa gestion scrupuleuse des moyens financiers. Les délégués prennent connaissance de l'état d'avancement des travaux de mise en ouvre des mesures de stabilisation budgétaire après le $1^{\mathrm{er}}$ semestre 2018.

\section{Elections et élections de confirmation}

\subsection{Confirmation des délégués nommés à l'AD}

L'art. 36a des Statuts de la FMH prévoit que les délégués ordinaires ainsi que les délégués suppléants nommés à l'Assemblée des délégués soient confirmés par la Chambre médicale. Seuls sont éligibles les délégués à la Chambre médicale ayant le droit de vote ou de proposition.

Proposition:

Les organisations délégantes VEDAG et CMPR proposent à la Chambre médicale de confirmer les nouveaux délégués suivants à l'Assemblée des délégués de la FMH dès le 25 octobre 2018:

\section{VEDAG}

Jusqu'ici: Beat GAFNER, Niederscherli

Nouveau: Esther S. HILFIKER, Berne

CMPR

Jusqu'ici: Marc MÜLLER, Grindelwald Franziska ZOGG-HARNISCHBERG, Zoug

Nouveau: Heidi ZINGGELER FUHRER, Coire Philippe LUCHSINGER, Affoltern am Albis

Décision:

Les délégués sont confirmés par 110 oui, 3 non et 0 abstention.

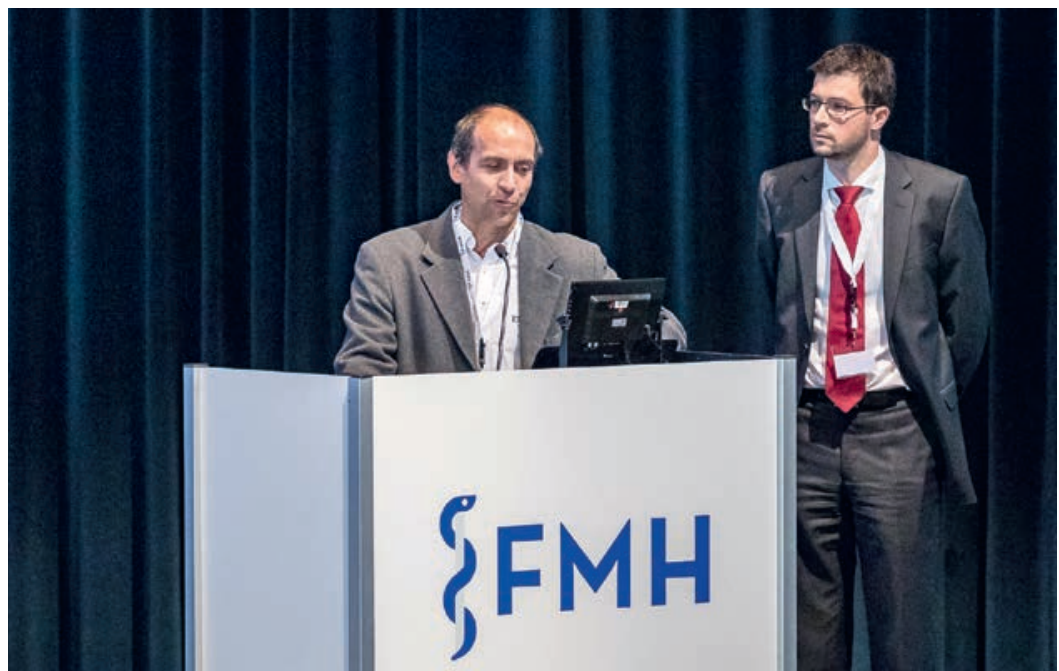

Le Dr German Clénin, président de la Société suisse de médecine du sport, tient un exposé sur l'encadrement médical des sportifs. A sa droite, Julien Duruz, juriste à la FMH.

\subsection{Confirmation des délégués suppléants} nommés à l'AD

L’art. 36a des Statuts de la FMH prévoit que les délégués ordinaires ainsi que les délégués suppléants nommés à l'Assemblée des délégués soient confirmés par la Chambre médicale. Seuls sont éligibles les délégués à la Chambre médicale ayant le droit de vote ou de proposition.

Proposition:

Les organisations délégantes VEDAG, CMPR, SFSM proposent à la Chambre médicale de confirmer les nouveaux délégués suppléants à l'Assemblée des délégués de la FMH dès le 25 octobre 2018:

\section{VEDAG}

Jusqu'ici: vacant

Nouveau: Lukas MEIER, Erlinsbach

CMPR

Jusqu'ici: Philippe LUCHSINGER, Affoltern am Albis

Nouveau: Franziska ZOGG-HARNISCHBERG, Zoug SFSM

Jusqu'ici: vacant

Nouveau: Tobias EHMANN, Horw

Décision:

Les délégués suppléants sont confirmés par 110 oui, 0 non et 3 abstentions.

\section{Modifications des Statuts, du Règlement d'exécution et du Code de déontologie}

\subsection{Modification du Code de déontologie de la FMH}

\subsubsection{Modification de l'annexe 5: directive pour la prise en charge médicale des sportifs} Jürg Schlup/président de la FMH accueille le Dr méd. German Clénin/président de la SSMS et l'avocat Julien Duruz/Service juridique de la FMH. Me Duruz attire l'attention sur les changements de l'annexe 5. La Chambre médicale a adopté l'annexe 5 du Code de déontologie en avril 2002. Cette annexe traite d'un thème global qui concerne les principes généraux régissant la prise en charge des sportifs et de la question spécifique du dopage. Depuis 2002, le droit du sport a fortement évolué, tout particulièrement en ce qui concerne la lutte antidopage. Une nouvelle loi fédérale sur l'encouragement du sport et de l'activité physique (LESp; RS 415.0) est notamment entrée en vigueur en 2012 et la réglementation sportive privée a connu de nombreux développements sous l'impulsion de l'Agence mondiale antidopage (AMA). L'annexe 5 du Code de déontologie de la FMH sur la prise en charge médicale des sportifs est actuellement dépassée et doit être adaptée pour inté- 
grer le droit fédéral et être compatible avec la réglementation privée. Le projet de révision a été élaboré en collaboration avec la SSMS et la Fondation Antidoping Suisse qui est le centre de compétence suisse de lutte antidopage. Le projet a fait l'objet d'une procédure de consultation auprès des organisations représentées à la Chambre médicale.

Les principales modifications concernent essentiellement la lutte antidopage. Les principes relatifs à la médecine sportive ont subi des modifications d'ordre formel. L'annexe essaie d'adopter une approche globale du dopage, du point de vue de l'éthique professionnelle, et d'interdire toute forme de dopage de la part du corps médical. Le Code de déontologie irait ainsi plus loin que le droit fédéral ou le droit sportif dans certains domaines: p. ex. le médecin qui se dope lui-même pour participer à une compétition n'est pénalement pas punissable, mais pourrait être sanctionné pour violation du Code de déontologie et de son annexe 5. Il n'est en effet pas justifiable, du point de vue de l'éthique professionnelle, qu'un médecin mette à profit ses connaissances médicales pour fausser la compétition à laquelle il participe.

La révision de l'annexe 5 offrira non seulement une source d'information solide au corps médical et aux instances de déontologie en matière de lutte antidopage, mais elle pourrait également servir d'outil pour les tribunaux pénaux et civils ainsi que les autorités de surveillance, lorsqu'ils sont amenés à définir/interpréter les devoirs professionnels en lien avec le dopage.

German Clénin/président de la SSMS expose le point de vue de la SSMS en présentant, sur la base de trois cas concrets, les clarifications que les médecins doivent effectuer avant de prescrire des médicaments.

La SSMS a été très tôt impliquée dans la révision de l'annexe 5 du Code de déontologie. Le Comité de la SSMS est favorable à l'adoption de la nouvelle version remaniée "Prise en charge médicale des sportifs».

Pour en savoir plus:

- SSMS: https://sgsm.ch/fr/

- Fondation Antidoping Suisse: https://www. antidoping.ch/fr/

- Base de données sur les médicaments: https://www. antidoping.ch/fr/medecine-substances-etmethodes/medicaments-suisse-interrogation

Après les débats, le président remercie les deux intervenants pour leur présentation et le travail fourni. Il prend congé de German Clénin.

Comme il s'agit de deux propositions, les délégués sont d'accord de procéder à un vote commun.
Proposition du Comité central:

La Chambre médicale:

a. approuve la modification de l'art. 33bis du Code de déontologie de la FMH proposée en annexe;

b. approuve la révision de l'annexe 5 au Code de déontologie de la FMH relative à la prise en charge médicale des sportifs proposée en annexe.

Décision:

La proposition est adoptée par 152 oui, 3 non et 3 abstentions.

\subsubsection{Modification des directives de l'ASSM} "Attitude face à la fin de vie et à la mort»

Jürg Schlup/président de la FMH rappelle en introduction que le Comité central soutient les nouvelles directives à l'exception du sous-chapitre 6.2.1. La notion de "souffrance insupportable» est une notion juridique imprécise qui place les médecins traitants face à des problèmes et qui crée une situation d'insécurité juridique. Le Comité central est favorable à un élargissement de la réglementation mais sur une base juridique sûre. Pour cela, il a formulé un projet et mené des entretiens avec l'ASSM en mars et en juillet 2018.

Le président accueille le Prof. Christian Kind qui, en tant que président de la sous-commission de la Commission Centrale d'Ethique, a élaboré les directives de l'ASSM en question. L'ASSM a procédé à une refonte complète de ses directives "Prise en charge des patientes et patients en fin de vie» et adopté un projet sous le titre "Attitude face à la fin de vie et à la mort» qu'elle a ensuite mis en consultation. L'ASSM a adopté les nouvelles directives lors de sa séance de mai 2018.

Le Prof. Kind souligne que le thème central de ces nouvelles directives n'est en aucun cas l'assistance au suicide ${ }^{1}$. Contrairement à la version précédente, les directives ne se limitent plus à la fin de vie du patient. Elles aident les médecins à aborder avec le patient la fin de vie et la mort si une maladie probablement mortelle est diagnostiquée. Elles réglementent l'admissibilité et la procédure de la sédation palliative ainsi que l'attitude à adopter face aux patients souhaitant mourir par un renoncement volontaire à toute nourriture solide et liquide. Par ailleurs, elles réglementent désormais aussi l'assistance au suicide pour les patients dont la fin de vie n'est pas proche. Dans les nouvelles directives, le dialogue sur la fin de vie et la mort est au centre des préoccupations et se fonde sur l'idée des soins palliatifs. Le Prof. Kind rappelle les conditions de l'admissibilité à l'assistance au suicide. La capacité de discernement du patient doit être documentée avec précision. En cas de maladie psychique, de démence ou d'un autre état fréquemment associé à une incapacité de discer- 
nement, cette dernière doit être évaluée par un médecin spécialiste. Le désir de mourir du patient doit être mûrement réfléchi, ne pas résulter d'une pression extérieure et être persistant. Une tierce personne indépendante est consultée pour dire si ces conditions sont remplies.

Les opposants aux nouvelles directives demandent la suppression du critère de "souffrance insupportable» et son remplacement par la condition d'être en présence d'une maladie mortelle non guérissable. Pour l'ASSM, ce n'est pas une voie appropriée pour réglementer l'aide au suicide. Si la notion de «maladie mortelle» est objective, elle reste difficile à délimiter avec précision. Les opposants critiquent également que les directives favorisent le respect absolu du libre arbitre du patient au détriment du caractère empathique de l'action du médecin sur le bien-être du patient. Cela n'est pas vrai. L'autodétermination d'un patient capable de discernement doit absolument être respectée. Même si un patient l'exige, le médecin ne peut rien entreprendre qui nuit au bien-être du patient. Avec l'aide au suicide, la principale difficulté réside dans le fait que les médecins, les éthiciens mais aussi le grand public ont en Suisse des avis partagés quant à savoir si elle contribue ou non au bien du patient. C'est pourquoi l'ASSM a décidé de tenir compte de cette réalité en confiant l'assistance au suicide en tant qu'action médicale controversée à la décision du médecin prise selon son âme et conscience. Or l'aide au suicide n'est pas considérée comme une action médicale généralement acceptée et ne peut donc pas, à ce titre, être proposée au patient sur l'initiative du médecin. Le souhait doit provenir du patient capable de discernement. Le médecin est censé appréhender le désir de mourir avec empathie et rechercher des options thérapeutiques ou d'autres offres d'aide et de soutien en vue de soulager la souffrance. Ce n'est que si cette démarche échoue que la demande d'aide au suicide sera estimée acceptable. Enfin, les opposants critiquent également que les nouvelles directives compromettraient la prévention du suicide.

En résumé, les directives confèrent aux médecins traitants la liberté d'agir selon leur propre éthique personnelle. Tout médecin qui n'envisage pas d'accompagner un suicide assisté n'est pas tenu de le faire. Il doit néanmoins informer le patient qu'il ne peut pas y être contraint. Si un médecin est fondamentalement favorable à l'aide au suicide mais, dans certains cas, ne parvient pas à comprendre la souffrance d'un patient spécifique, il devra rejeter sa demande. En revanche, s'il a vérifié que toutes les conditions sont remplies dans le cas concret et s'il est convaincu d'agir pour le bien du patient, il pourra s'appuyer sur ces directives qui lui apporteront alors une plus grande sécurité. Dans les autres cas, ces dernières n'apportent aucune modification en matière d'assistance au suicide. Pour le Prof. Kind, il est peu probable qu'un rejet de la Chambre médicale incite le législateur à réglementer l'aide au suicide dans une loi, tous les efforts déployés jusqu'ici n'ayant pas abouti. Du point de vue éthique, il ne serait pas non plus souhaitable d'inscrire dans la loi la réglementation détaillée d'une problématique aussi complexe et en grande partie intime et personnelle. Il est, selon lui, tout aussi improbable que l'application des nouvelles directives ait un impact important sur la fréquence des cas d'assistance au suicide. Toute personne désireuse de bénéficier de l'aide au suicide en Suisse ces dernières années a pu en règle générale l'obtenir. Un maintien des anciennes directives de l'ASSM dans le Code de déontologie reviendrait à faire coexister deux directives différentes, ce qui entraînerait une nouvelle insécurité juridique notamment pour les médecins disposés à accompagner une aide au suicide.

Jürg Schlup/président de la FMH fait observer qu'en cas de rejet des nouvelles directives de l'ASSM, les anciennes directives qui font aujourd'hui déjà partie intégrante du Code de déontologie de la FMH continueraient d'être valables. Après quelques questions, le président remercie le Prof. Kind de ses explications.

Le Dr Michael Barnikol/juriste du Service juridique de la $F M H$ ouvre ensuite la discussion. Il précise que, selon lui, de nombreuses organisations de médecins se sont exprimées dans le cadre de la procédure de consultation surtout sur les directives en général, mais beaucoup moins sur la question de l'aide au suicide en particulier (sous-chapitre 6.2.1). L'avis du Prof. Kind, selon lequel la grande majorité des sociétés de médecins se serait exprimée explicitement en faveur de la réglementation de l'aide au suicide dans les nouvelles directives de l'ASSM n'est d'après lui pas tout à fait correct. Il aborde les différents contenus et questions que cette révision soulève. En cas d'approbation par la Chambre médicale, cette réglementation serait reprise dans le Code de déontologie de la FMH et deviendrait donc contraignante. En cas de non-respect, les commissions de déontologie cantonales pourraient prononcer des sanctions. L'applicabilité de l'aide au suicide selon le chiffre 6.2.1 des nouvelles directives va beaucoup plus loin que l'ancienne réglementation. La notion de "souffrance insupportable» est imprécise et difficilement vérifiable pour le médecin, ce qui représente un facteur d'incertitude important pour les médecins concernés. Il est de l'intérêt du corps médical de disposer de critères clairement formulés. Avec les nouvelles directives, l'ASSM voulait répondre à une évolution sociétale selon laquelle le souhait d'assistance au suicide ne 
se limite plus à la fin de vie du patient. Mais sur le plan juridique, la réglementation proposée n'est pas équilibrée.

Philippe Luchsinger/SSMIG explique que pour la SSMIG, les directives sont claires et qu'en cas de rejet de la proposition de l'Assemblée des délégués, les nouvelles directives de l'ASSM devront être reprises dans le Code de déontologie. Aussi différents que sont les parcours de vie des êtres humains, aussi diverses sont leurs façons de vouloir mourir. Le patient doit être capable de discernement, son désir de mourir persistant, ne pas résulter d'une pression extérieure et être motivé par une souffrance que le patient juge insupportable et pour laquelle d'autres options thérapeutiques ont échoué. Et si possible, l'entourage du patient doit être impliqué dans ce processus. Des incertitudes subsisteront toujours au quotidien. Chaque médecin doit avoir la possibilité d'accomplir, selon sa propre conception et non celle imposée par des tiers, sa principale activité, à savoir accompagner, encadrer et assister ses patientes et ses patients dans toutes les situations de la vie, dont la fin de vie et la mort, même en cas de suicide assisté. Pierre Vallon/président de l'Assemblée des délégués expose une nouvelle fois les raisons qui ont motivé la proposition l'Assemblée des délégués. Dans sa prise de position, le Comité central a pointé du doigt le critère trop indéterminé de l'assistance au suicide qui serait autorisée dès la présence de "souffrance insupportable» du patient et non plus uniquement en fin de vie. Il propose de limiter le droit à l'assistance au suicide aux patients atteints d'une maladie mortelle, dont l'état ne s'améliorera pas avec un traitement médical approprié. Après avoir longuement débattu sur la reprise de ces directives dans le Code de déontologie,

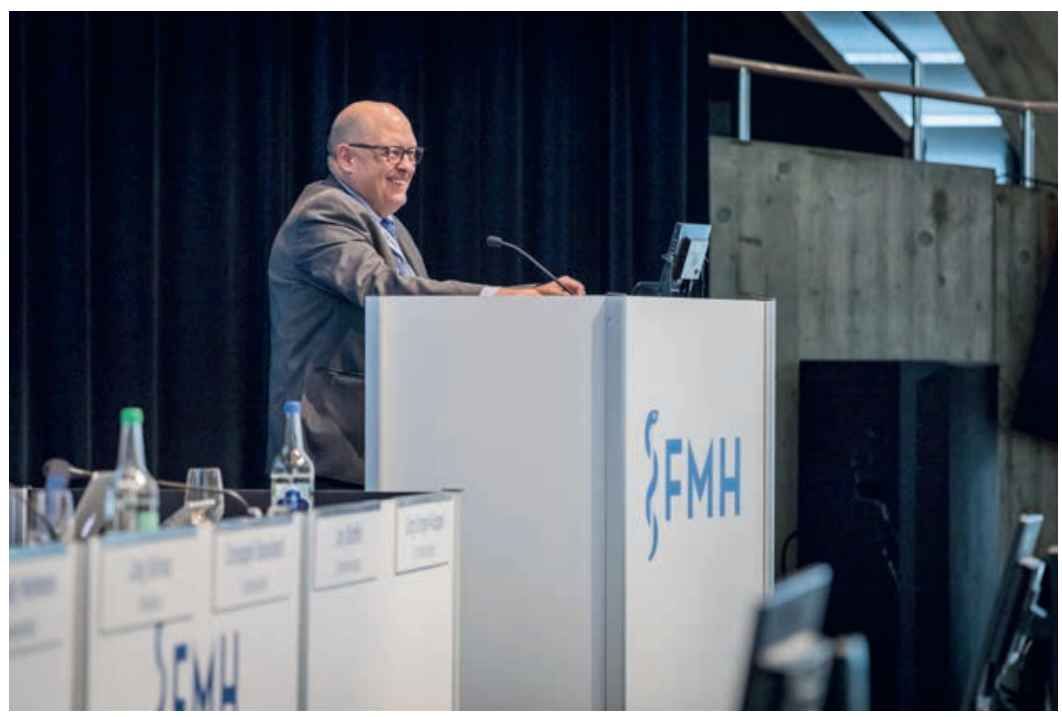

Le président de l'Assemblée des délégués (AD), Pierre Vallon, présente la proposition de I'AD.
l'Assemblée des délégués a finalement décidé de les rejeter. Elle motive ce rejet par le fait que le corps médical serait seul à porter la responsabilité de l'assistance au suicide et elle s'inquiète de l'applicabilité correcte des nouvelles dispositions aussi bien du point de vue sociétal que juridique. L'Assemblée des délégués ne veut reprendre les nouvelles directives dans le Code de déontologie de la FMH que lorsque l'ASSM aura remplacé la notion de "souffrance insupportable» par une notion juridique clairement définie.

Après une longue liste d'orateurs, le président dépose une motion d'ordre.

Proposition du Comité central:

La liste des orateurs s'achèvera après les trois prochaines interventions.

\section{Décision:}

La motion d'ordre est approuvée à la grande majorité.

Au terme d'une discussion intense, une majorité considère que la reprise des nouvelles directives de l'ASSM engendrerait une grande incertitude juridique. La FMH souhaite que le Code de déontologie contienne des directives claires à l'intention des médecins et des patientes et patients, basées sur des critères objectivement vérifiables.

La FMH pourrait être ouverte à une plus grande libéralisation et propose que l'assistance au suicide se limite aux patients atteints d'une maladie mortelle, dont l'état ne s'améliorera pas avec un traitement médical approprié. Dans ce cas, le médecin peut poser un diagnostic fiable.

Jürg Schlup/président de la FMH propose de confronter les deux propositions.

Proposition du Comité central:

La proposition de l'Assemblée des délégués est confrontée à la proposition de la SSMIG.

\section{Décision:}

La proposition est adoptée à une grande majorité, 6 non et 5 abstentions.

Proposition de l'Assemblée des délégués:

La Chambre médicale décide que les directives de l'ASSM "Attitude face à la fin de vie et à la mort» ne soient pas reprises dans le Code de déontologie de la FMH tant que la notion de "souffrance insupportable» au chapitre 6.2.1 des directives n'a pas été remplacée par une notion juridique clairement définie.

Proposition de la SSMIG:

Les nouvelles directives 2018 de l'ASSM «Attitude face à la fin de vie et à la mort» doivent être reprises dans le Code de déontologie de la FMH. 


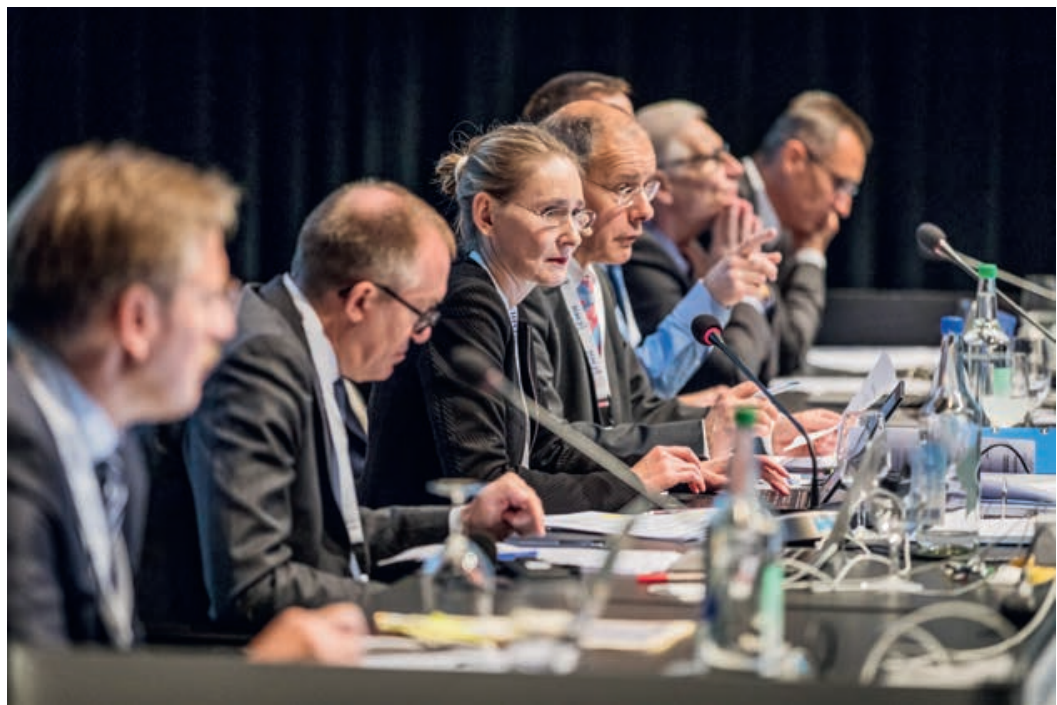

En fonction depuis le $1^{\text {er }}$ octobre, Ursina Pally Hofmann, secrétaire générale de la FMH et docteur en droit. jamais eu l'intention de blesser quelqu'un. La Suisse a besoin d'un plus grand nombre de médecins de famille et de l'enfance, raison pour laquelle l'Assemblée des délégués de mfe a donné un mandat dans ce sens. Le but était de mettre l'accent sur l'attrait de la médecine de famille. La campagne s'adressait aux responsables politiques, aux étudiants en médecine, aux médias et au public. L'association mfe a choisi une approche non conventionnelle pour surprendre le public-cible. Le message essentiel était que $94 \%$ des problèmes de santé peuvent être résolus par un médecin de famille ou un pédiatre. Cette affirmation est scientifiquement prouvée par une étude de l'Institut pour la médecine de famille de Zurich. En tant que président de mfe, il regrette que certaines personnes aient été blessées par cette campagne et s'en excuse; mfe supprimera ces vidéos sur internet.

Josef Brandenberg/président de la fmCh remercie tous les membres de mfe qui prennent leurs distances avec cette action. Si les responsables de mfe effectuent les suppressions appropriées sur le site internet, la situation se calmera parmi les membres de la fmCh. Cela dit, l'association faîtière considère qu'il est important de clarifier la question des sanctions envers les organisations médicales et demande la révision des articles correspondants du Code de déontologie de la FMH.

Ursina Pally Hofmann/secrétaire générale et cheffe du Service juridique de la FMH attire l'attention sur les aspects juridiques. Les modifications proposées par la fmCh équivalent à un changement de système qui aurait des répercussions importantes à long terme et seraient irréalistes. Les règles actuelles du Code de déontologie s'appliquent aux médecins en tant que personnes physiques. Selon les Statuts de la FMH, les membres s'engagent à respecter lesdits Statuts ainsi que le Code de déontologie. Il en va autrement pour les organisations médicales. Dans ce cas, il ne s'agit pas de médecins en exercice qui soignent des patients, mais de représentation d'intérêts. Les organisations médicales de la FMH ne s'engagent pas non plus à respecter le Code de déontologie, mais seulement les Statuts. Un changement ne peut être initié que par une révision des Statuts. Ce qui demande toutefois un remaniement systématique et bien pensé, incluant un changement du droit de procédure.

Après quelques interventions, Felix Kurth/ASMAC dépose une motion d'ordre visant à ce que l'on vote maintenant sur la proposition.

Motion ASMAC:

Fin des interventions et exécution du vote. Décision:

La proposition est acceptée à une large majorité. 
Proposition de la fmCh:

Le Code de déontologie de la FMH doit être complété de la façon suivante:

Art. 23 Collégialité, critiques inadmissibles

Les médecins (nouveau) et les organisations médicales (organisations faîtières, sociétés cantonales de médecine, sociétés de discipline médicale, associations professionnelles de médecins) entretiennent entre eux des rapports confraternels empreints d'honnêteté et de courtoisie.

Le médecin s'interdit tout propos ou attitude qui puisse discréditer un confrère.

(Nouveau) Il est interdit d'adopter de façon injustifiée un comportement blessant envers les organisations médicales, les disciplines médicales, les regroupements professionnels de médecins et leurs membres. Appelé à donner son appréciation devant des tiers sur des actes, comportements ou propos de confrères, le médecin fait preuve de retenue et d'objectivité.

(Nouveau) Les organisations médicales ont vis-à-vis de tiers des propos toujours factuels et objectifs sur les activités des autres organisations médicales et de leurs membres.

Art. 30 Règlement de litiges

Le médecin (nouveau) ou l'organisation médicale s'efforce de régler personnellement ou avec l'aide de tiers tout litige qui l'oppose à un confrère ou à une organisation médicale et qui trouve son origine dans une infraction au Code de déontologie. En cas d'échec de la tentative de conciliation, le litige est porté devant l'instance professionnelle compétente.

Annexe 2 au Code de déontologie de la FMH: Directives pour l'information et la publicité

2. Publicité illicite (Code de déontologie, art. 20 al. 2

2.3. L'information nuit à la réputation de la profession médicale, en particulier lorsqu'elle:

- établit des comparaisons discréditant des confrères, rabaissant p. ex. leur activité ou leurs méthodes médicales;

- (nouveau) dénigre les activités d'autres organisations médicales, disciplines médicales, regroupements professionnels et de leurs membres.

Annexe 3 au Code de déontologie de la FMH: Directives concernant l'activité médiatique du médecin (nouveau) et des organisations médicales 2. Le médecin ne doit pas mettre exagérément en évidence son activité médicale. Il se garde de critiquer les méthodes thérapeutiques de confrères (nouveau) et d'organisations médicales ou de lancer une polémique à leur égard.
Décision:

La proposition est rejetée par 105 non contre 3 oui, avec 10 abstentions.

Pierre Vallon/président de l'Assemblée des délégués suggère de discuter cette question en profondeur et avec suffisamment de temps lors de l'Assemblée des délégués, qui est, à son avis, l'organe correct pour cela.

Jürg Schlup/président de la FMH soutient la proposition de Pierre Vallon/président de l'Assemblée des délégués. Il recommande à la fmCh de planifier les étapes suivantes, tout en tenant compte des objections du Service juridique. La FMH ne dispose aujourd'hui d'aucune base légale pour prendre des mesures contre les organisations médicales affiliées.

Pour Josef Brandenberg/président de la fmCh, la suite de la procédure dépendra du fait que mfe, à la suite des interventions de ce jour, supprime les vidéos sur internet.

\section{Données structurelles des cabinets médicaux et des centres ambulatoires (MAS)}

Christoph Bosshard/vice-président de la FMH et responsable du département Données, démographie et qualité revient sur la première collecte de données, qui s'est achevée en juin 2017. Celle-ci servait à des fins purement statistiques. L'art. 30 OAMal, l'art. 50a LAMal et le règlement de traitement ne s'appliquaient pas encore à cette collecte. La FMH a ensuite procédé, en collaboration avec l'Office fédéral de la statistique (OFS), à une plausibilisation des données. L'OFS a publié cette évaluation le 10 avril 2018. Les résultats de l'enquête MAS 2015 sont d'une importance majeure et d'un grand intérêt pour la FMH et le corps médical. Lélaboration de statistiques sur les soins ambulatoires devrait contribuer à la mise en place d'un système intégré d'information statistique sur la santé.

Le deuxième relevé, concernant les données de l'année 2017, débutera le 12 novembre 2018. Hormis son utilisation dans un but statistique, il servira désormais aussi à des fins de contrôle. Pour la première fois, le but de surveillance légale au sens de l'art 59 LAMal et de l'art. 30 de l'ordonnance qui s'y rapporte ainsi que du règlement de traitement est applicable. L'OFS ne prévoit aucune sanction envers les fournisseurs de prestations qui ne participent pas à ce relevé. Les données en registrées lors du relevé 2015 peuvent être importées. Christoph Bosshard accueille Marco D'Angelo/vice-directeur et chef de la division Santé et affaires sociales de l'OFS, lequel présente quelques résultats de la première enquête et informe des prochaines étapes. Le premier 
relevé s'est achevé avec succès au milieu de l'année 2017. Dans le cadre de ce relevé, 18723 entreprises ont été interrogées. Environ trois quarts des cabinets médicaux et centres ambulatoires contactés ont fourni des données sur leur situation. Près de 7000 cabinets médicaux et centres ambulatoires, soit la moitié des entreprises appartenant à l'ensemble corrigé, ont rempli le questionnaire et fourni des données. Les premiers résultats ont été publiés en avril 2018. Le deuxième relevé sera effectué aussi dans un but de surveillance selon le mandat légal de l'art. 59a LAMal. Le règlement de traitement valable pour les données 2017 est entré en vigueur le 20 mars 2017. Selon ce règlement, les données fournies à des fins de surveillance et de statistique par les cabinets médicaux et les centres ambulatoires sont anonymisées. Le cabinet médical et le médecin particulier ne sont identifiables à aucun moment. Pour la deuxième enquête, l'OFS a optimisé le questionnaire et a de nouveau mis en place une hotline.

Christoph Bosshard/vice-président de la FMH et responsable du département Données, démographie et qualité qualifie la collaboration avec l'OFS de constructive et fructueuse et remercie Marco D'Angelo pour sa présence aujourd'hui. Pour toute information, le département Données, démographie et qualité reste volontiers à la disposition des membres.

\section{Proposition SMVS: «Financement moniste»}

La SMVS et la SMSR ont déposé des propositions à ce propos, respectivement le 29 septembre et le 9 octobre 2018. Jürg Schlup/président de la FMH rappelle la décision de l'Assemblée des délégués du 29 juin 2017. A cette occasion, l'Assemblée des délégués avait approuvé à l'unanimité, sans opposition ni abstention, un financement uniforme des prestations médicales hospitalières et ambulatoires. Elle a en outre donné sa préférence à ce que l'argent des cantons soit remis aux assureurs par l'intermédiaire d'une institution LAMal commune.

Monique Lehky Hagen/présidente de la SMVS aborde la problématique du financement moniste. A l'origine, il était en fait prévu de transférer les coûts du secteur hospitalier vers le secteur ambulatoire. Aujourd'hui, force est de constater que les buts du financement uniforme ne sont pas atteints. Au contraire, le souhait de pilotage de l'ensemble du secteur ambulatoire, exprimé par différents cantons, risque de conduire à la suppression de l'obligation de contracter. On peut craindre qu'un tel pilotage se fasse au détriment du secteur ambulatoire extrahospitalier. De plus, ce projet de loi augmentera la pression politique sur toutes les négo- ciations tarifaires ambulatoires cantonales. L'adaptation proposée de la LAMal aboutira à une charge administrative encore plus grande. Le but de cette proposition est d'attirer l'attention sur cette situation, afin que la FMH corrige au plus tôt la trajectoire.

Philippe Eggimann/président de la SMSR explique que la position des cantons a changé par rapport au moment où l'Assemblée des délégués a décidé de soutenir le financement moniste. Dans sa première proposition, la SMSR demande à la FMH de lier le maintien de son soutien au financement moniste à la condition que les cantons retirent leur demande d'exiger une planification de l'ambulatoire des cabinets médicaux indépendants. Le financement moniste actuellement proposé prévoit que les cantons doivent supporter au moins $25,5 \%$ des coûts qui restent à la charge des assureurs après déduction des franchises et des quotes-parts assumées par les assurés.

La deuxième proposition de la SMSR vise un financement différencié. Elle demande que le financement moniste soit restreint aux prestations découlant du transfert de l'hospitalier vers l'ambulatoire, en particulier aux prestations dont la Confédération et/ou les cantons ne garantissent le remboursement que si elles sont effectuées en ambulatoire.

Josef Widler/président de l'AGZ fait observer qu'entretemps la situation a changé. A son avis, avec les trois propositions présentées, la FMH court le risque de limiter son propre pouvoir de négociation. La proposition déposée aujourd'hui par l'AGZ demande que la FMH soutienne le financement moniste. $\mathrm{Si}$, lors de la mise en œuvre, les cantons en viennent à piloter le secteur ambulatoire, il est indispensable que les sociétés cantonales de médecine soient impliquées de plein droit dans la planification, sous la forme d'un partenariat. Josef Widler/président de l'AGZ invite les délégués à soutenir la proposition de l'AGZ et demande aux délégués de la SMVS et de la SMSR s'ils retirent leurs propositions.

Pierre Vallon/président de l'Assemblée des délégués rappelle encore une fois la décision de l'Assemblée des délégués du 29 juin 2017. Les questions soulevées aujourd'hui sont complexes et nécessitent une discussion plus approfondie. Il propose donc de traiter ce sujet en profondeur lors de la prochaine Assemblée des délégués.

Jürg Schlup/président de la FMH soutient la proposition de Pierre Vallon/président de l'Assemblée des délégués. Il est déjà $18 \mathrm{~h}$. Or, selon la décision de la Chambre médicale de ce jour, plus aucune décision ne peut être prise. Il invite donc les deux délégués à déposer leurs propositions en temps utile pour la prochaine Assemblée des délégués. 


\section{Rénovation complète du siège de la FMH à Berne}

Christoph Bosshard/vice-président de la FMH rappelle la décision de la Chambre médicale du 26 octobre 2017, où les délégués se sont prononcés en faveur d'une rénovation complète du bâtiment de l'Elfenstrasse. Cette décision avait été précédée d'un long débat qui avait également porté sur différentes variantes allant de la vente et d'un nouvel achat à la rénovation complète du bâtiment et à sa réutilisation, en passant par la location et l'affermage.

En application de la décision de la Chambre médicale, le Comité central a mis sur pied une instance responsable du projet, à laquelle ont été confiées les tâches relatives au développement de ce projet. Sur la base de l'analyse de faisabilité sur laquelle s'est fondée la décision de la Chambre médicale, le Comité central a mené des études approfondies dans le domaine des matériaux de construction, y compris les polluants à risque. Afin de concrétiser la planification, une analyse détaillée des besoins a également été effectuée, portant non seulement sur le bâtiment, mais sur l'ensemble de la propriété. Ces travaux d'analyse et de planification ont été achevés en été. L'avant-projet qui en a résulté a été approuvé par le Comité central le 22 août 2018. Cet avant-projet est, d'une part, associé à une précision de planification de plus ou moins $20 \%$, d'autre part, il montre, sur la base d'estimations plus prudentes, qu'il faut éventuellement compter avec des coûts légèrement plus élevés que la marge approuvée. Vu la phase où se trouve le projet, la question du lieu de remplacement durant la phase de rénovation n'a pas encore été concrétisée. Le marché est cependant relativement dynamique et favorable.

Le Comité central approuvera la planification détaillée définitive de la rénovation au printemps 2019. Ensuite, la demande de permis de construire sera préparée. Les travaux de rénovation ne débuteront pas avant novembre 2019. Le 9 mai 2019, la Chambre médicale sera informée de la suite du projet et devra se prononcer sur des propositions motivées, pour autant que le cadre budgétaire ne puisse être respecté.

\section{Informations du Comité central, du Secrétariat général, de l'ISFM et des divisions}

Les délégués ont reçu à l'avance et par écrit les informations du Comité central, du Secrétariat général, de l'ISFM et des divisions.

Yvonne Gilli/membre du Comité central et responsable du département Numérisation et eHealth ajoute un bref résumé oral de la mise en œuvre des mesures concer- nant le Règlement général européen sur la protection des données (RGPD). Dans certaines conditions, le RGPD a des incidences sur les hôpitaux et les cabinets médicaux. Les entreprises suisses sont en effet touchées par l'effet extraterritorial du RGPD après son entrée en vigueur obligatoire pour les Etats membres européens le 25 mai 2018.

Le Comité central a proposé à la Chambre médicale du 26 octobre 2017 que tous les organes de la FMH réexaminent leur organisation et leurs processus, pour vérifier si les exigences minimales de la révision de la loi sur la protection des données sont remplies, et qu'ils mettent en œuvre rapidement les éventuelles mesures correctives nécessaires. Les délégués ne sont pas entrés en matière sur cette proposition.

Depuis l'entrée en vigueur du RGPD de l'UE en mai 2018, une avalanche de questions à ce sujet sont parvenues au département. Bien que seuls quelques cabinets médicaux et hôpitaux soient concernés par ce nouveau règlement, le département Numérisation et eHealth a élaboré un document de "questions et réponses», qui traite de l'applicabilité et des effets du règlement. Afin de réduire au minimum les risques liés à l'applicabilité du RGPD, le département a en plus élaboré une Déclaration de protection des données en collaboration avec HIN SA. Cette déclaration s'adresse essentiellement aux médecins qui publient des informations ou proposent des services sur leur propre site internet. Pour en savoir plus: https://www.fmh.ch/fr/politique themes/ehealth/protection-des-donnees.html.

\section{Divers}

Le Dr Angelo Canova/AGZ attire l'attention sur les multiples mandats des délégués. Afin d'assurer aussi à l'avenir une formation pluraliste de l'opinion, il propose que les candidats rendent publiques leurs différentes fonctions lors d'élections.

\section{Dates de la Chambre médicale en 2019}

Jeudi 9 mai 2019 (et non le 2 mai 2019 comme prévu initialement)

Jeudi 31 octobre 2019

\section{Dates de la Chambre médicale en 2020}

Jeudi 14 mai 2020

Jeudi 29 octobre 2020

Jürg Schlup/président de la FMH met fin à la séance et prend congé des délégués. Il les remercie pour leur participation active et constructive. Ses remerciements vont également à l'équipe du Secrétariat général qui a de nouveau assuré une excellente organisation de la Chambre médicale. Il souhaite à tous un bon retour. 


\section{Glossaire}

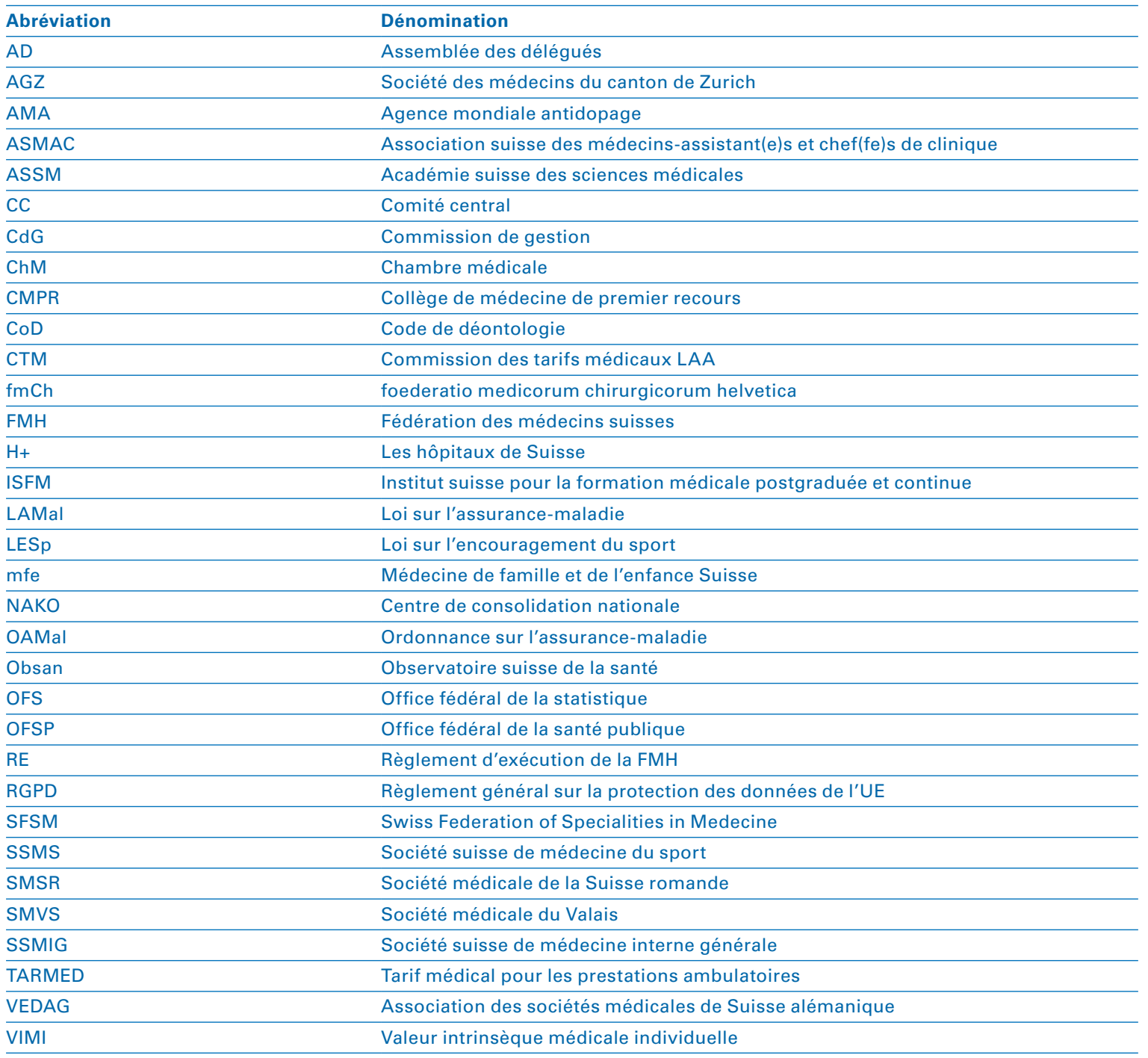

